\title{
Meta-memória e Auto-eficácia: \\ Um Estudo de Validação de Instrumentos de Pesquisa sobre Memória e Envelhecimento
}

\author{
Mônica Sanches Yassuda ${ }^{12}$ \\ Valéria Bellini Lasca \\ Anita Liberalesso Neri \\ Universidade Estadual de Campinas
}

\begin{abstract}
Resumo
A literatura cognitiva documenta em inúmeros relatos a existência de diferenças significativas entre o desempenho de jovens e idosos em tarefas de memória episódica. Pesquisadores nas últimas décadas exploram possíveis explicações para esta discrepância. Uma das hipóteses em investigação é que talvez os idosos possuam percepções, atitudes e afetos diferentes relativos à memória, e que talvez seu desempenho seja afetado por estes fatores. Os conceitos de meta-memória e auto-eficácia têm sido empregados nas pesquisas sobre o envelhecimento da memória para a avaliação desta hipótese. Nesta pesquisa, 1 questionário de meta-memória (MIA), 1 questionário de auto-eficácia (MSEQ) e 4 versões de 2 tarefas de desempenho de memória episódica (4 listas de itens de supermercado e 4 histórias curtas) foram traduzidos para o português, adaptados à cultura brasileira e submetidos a testes de validação junto a 33 jovens e 27 idosos saudáveis brasileiros. Os resultados das análises realizadas sugerem que as versões em português destes instrumentos têm boas características psicométricas e são promissores para esta área de pesquisa no Brasil.

Palavras-chave: Meta-memória; auto-eficácia; memória episódica; envelhecimento.
\end{abstract}

\section{Metamemory and Self-efficacy: A Validation Study of Research Instruments in Memory Aging}

\begin{abstract}
The cognitive aging literature documents in numerous studies the existence of significant age differences between younger and older adults in tests of episodic memory. Researchers have been investigating possible explanations for such differences. One hypothesis being tested is whether older adults hold different perceptions, attitudes and feelings towards memory that could affect performance negatively. Metamemory and self-efficacy are concepts that have been used in the test of this hypothesis. In the present research, a metamemory questionnaire (MIA), a self-efficacy questionnaire (MSEQ) and 4 versions of 2 episodic memory tasks (4 grocery lists and 4 short stories) were translated into Portuguese, adapted to Brazilian culture and submitted to validity testing with 33 younger and 27 older healthy Brazilian adults. The results from the analyses suggest that the Portuguese versions of these instruments have good psychometric characteristics and seem promising for research in this field in Brazil.

Keywords: Metamemory; self-efficacy; episodic memory; aging.
\end{abstract}

O objetivo desse estudo é apresentar a versão em português assim como dados de validação de instrumentos de pesquisa já reconhecidos na literatura internacional, que podem ser úteis para o desenvolvimento da pesquisa sobre o envelhecimento da memória junto à população brasileira. A introdução a seguir apresenta os conceitos teóricos importantes para o estudo realizado assim como referências a estudos selecionados sobre o tema.

Nos últimos 50 anos, geriatras e gerontólogos têm pesquisado intensamente os efeitos do envelhecimento no funcionamento da memória humana. Diversas mudanças no funcionamento da memória já foram bem documentadas, e são esperadas durante o envelhecimento saudável (Abreu \& Xavier, 2001; Yassuda, 2002). Por exemplo, sabemos hoje que a velocidade do processamento das informações, importante a formação de novos traços de memória, é menor nos adultos mais velhos do que nos jovens. Isto é, em média, um senhor de 65 anos necessitará de mais tempo para processar (ler, compreender, memorizar) as informações da primeira página do jornal do que seu neto de

${ }^{1}$ Endereço para correspondência: Al. Piunas, 140, Aldeia da Serra, Barueri, SP, 06519 390. Fone: (11) 41922548; Fax. (11) 41924053.E-mail: yassuda@usp.br

${ }^{2}$ As autoras gostariam de agradecer a todos os voluntários que participaram da pesquisa e a FAPESP pelo apoio financeiro ao projeto.
20 anos. Sabemos também que o declínio que ocorre com a idade na memória para fatos, também chamada de memória episódica, é mais acentuado do que o declínio que ocorre na memória semântica, que usamos para materiais lingüísticos. Outro dado documentado e bem aceito pelos pesquisadores é que a memorização consciente, também chamada de memória explícita, é mais sensível aos efeitos do envelhecimento do que a memorização realizada inconscientemente (memória implícita).

Ao longo das últimas décadas, diferentes hipóteses foram elaboradas com o intuito de explicar o declínio no desempenho dos idosos em diversas tarefas de memória. Uma destas hipóteses, conhecida como a hipótese do desuso, aponta que o estilo de vida do idoso pode não favorecer o bom funcionamento da memória. Em função da aposentadoria e mudanças nos papéis sociais, os idosos podem se afastar da estimulação cognitiva necessária, o que levaria ao desuso das habilidades de memória. Outra hipótese aponta para o envelhecimento biológico cerebral, que pode ocasionar a perda de neurônios ou a redução das conexões entre eles, sendo responsável pela lentificação do processamento das informações. Outras hipóteses sugerem que o idoso tem um estilo cognitivo menos eficiente, pois tende a usar menos estratégias de memorização, tem dificuldade de inibir o processamento de informações irrelevantes e tem menor 
controle sobre sua atenção (para uma discussão detalhada sobre estas hipóteses, ver Salthouse, 1991).

Uma hipótese mais recente e que tem atraído a atenção dos pesquisadores é a idéia que o desempenho dos idosos em tarefas de memória pode também ser negativamente influenciado por suas atitudes e crenças a respeito de sua capacidade de memorizar. $\mathrm{Na}$ literatura cognitiva este conjunto de idéias e sentimentos a respeito da memória é chamado de meta-memória. Esta hipótese afirma que alterações particulares na meta-memória do idoso, como crenças mais negativas sobre a memória (Ex.: achar que o envelhecimento envolve perdas irreversíveis na memória) podem influenciar importantes variáveis moduladoras do desempenho, tais como esforço despendido, motivação e o uso de estratégias. Apesar do número de artigos publicados sobre este tema ser ainda limitado, algumas pesquisas sugerem que as crenças do memorizador podem influenciar a maneira como a tarefa é realizada, assim como o desempenho final (Berry \& West, 1983; Hertzog, McGuire \& Lineweaver, 1998; Welch \& West, 1995; West, Welch \& Thorn, 2001).

Como ilustração, podemos citar a pesquisa de Hertzog e colaboradores (1998). Adultos jovens e idosos responderam dois questionários sobre percepções de memória, realizaram um teste de memória episódica e o uso de estratégias mnemônicas durante o teste foi avaliado, entre outras medidas. Os resultados indicaram correlações significativas entre percepções de controle sobre a memória, o desempenho objetivo na tarefa de memorização e o uso de estratégias. Modelos matemáticos elaborados a partir das correlações entre as variáveis estudadas revelaram que parte dos efeitos do envelhecimento sobre o desempenho de memória é mediada por variáveis que fazem parte da meta-memória, como a auto-eficácia (definida abaixo) e o controle percebido.

As pesquisas realizadas a respeito da importância das crenças para a memória foram realizadas dentro de perspectivas teóricas diversas, tais como o estudo da meta-memória (Flavel, 1971), a teoria da auto-eficácia (Bandura, 1977), do foco de controle ou locus of control (Lachman, 1983), e também a teoria da atribuição (Abranson, Seligman \& Teasdale, 1978). Neste artigo, maior atenção será dada ao conceito de meta-memória e de auto-eficácia, para posteriormente apresentarmos dados sobre a adaptação e validação de um questionário de meta-memória e de auto-eficácia no Brasil.

\section{Meta-memória}

O termo meta-memória originalmente foi cunhado para se referir ao conhecimento objetivo de um indivíduo sobre os processos da memória, como por exemplo, o grau de dificuldade de uma determinada tarefa ou quais as estratégias apropriadas para realizá-la (Flavell, 1971; Flavell \& Wellman, 1977). Entretanto, esta definição mostrou-se limitada para o estudo do envelhecimento cognitivo, e atualmente o conceito de meta-memória inclui diversos aspectos, tais como: a) o conhecimento sobre os processos da memória (Ex.: quais tarefas de memória são fáceis e quais são difíceis); b) o monitoramento da memória (Ex.: a capacidade de uma pessoa avaliar se já estudou o suficiente para uma prova); c) sentimentos e emoções sobre a memória; e d) a auto-eficácia para memória, que poderia ser definida como o grau de certeza de um individuo sobre sua capacidade de realizar uma tarefa envolvendo memória. Atualmente, é bem aceita entre os pesquisadores a visão que a auto-eficácia seria uma das dimensões do conceito mais amplo da meta-memória.

A meta-memória tem sido estudada dentro de duas posturas metodológicas diferentes. Dentro da postura da psicometria, questionários foram desenvolvidos para captar os conhecimentos, as percepções e os sentimentos dos indivíduos sobre a memória, como o Metamemory in AdulthoodQuestionnaire (MIA-Dixon \& Hultsch, 1983a, 1983b) e o Memory Functioning Questionnaire (MFQ- Gilewski, Zelinski, Schaie \& Thompson, 1983). A outra perspectiva utilizou-se de previsões de desempenho, dentro dos paradigmas de feelings-ofknowing judgements-of-knowing. No primeiro, pergunta-se ao participante sobre a existência de um traço de memória após o estudo do material, e no segundo, pede-se para o participante prever qual será o seu desempenho em uma tarefa a ser realizada, antes do estudo do material (ver Lovelace, 1990).

O questionário MIA (Dixon \& Hultsch, 1983a, 1983b) foi utilizado em diversos estudos em países diferentes.Este questionário é composto por 108 questões que avaliam sete dimensões da meta-memória: a) Estratégia-conhecimento sobre estratégias ou utilização de estratégias de memória; b) Tarefaconhecimento de processos básicos de memória; c) Capacidadepercepções sobre sua própria capacidade de memorizar; d) Mudança- alterações percebidas na capacidade de memorizar com o envelhecimento; e) Ansiedade- estresse relacionado à situações de memorização; f) Metas- importância de ter bom desempenho em tarefas de memória; e g) Controle - percepção de controle sobre as habilidades de memória. Em estudos posteriores sobre as características psicométricas do questionário, Hertzog, Dixon, Schulenberg e Hultsch (1987) evidenciaram que estas sub-escalas podem compor dois fatores dentro do questionário. As sub-escalas Estratégia, Tarefa e Metas formam o fator Conhecimento, e as sub-escalas Capacidade, Controle e Mudança formam o fator Auto-Eficácia. A sub-escala Ansiedade tem suas questões correlacionadas com os dois fatores.

Pesquisas, realizadas com adultos jovens e idosos utilizando o MIA, revelaram que o fator Conhecimento permanece basicamente inalterado com o avançar da idade, enquanto o fator Auto-eficácia mostra-se bastante sensível ao envelhecimento. Particularmente, a sub-escala Mudança é a que apresenta maior correlação com a variável idade. Estes estudos revelaram que os idosos percebem declínios significativos em sua memória (resultados da subescala Mudança) e relatam menor percepção de controle sobre ela (resultados da sub-escala Controle). Os idosos também tendem a perceber-se com menor capacidade de memória (resultados da subescala Capacidade) (Hultsch, Hertzog, Dixon \& Davidson, 1988).

Um ponto que deve ser ressaltado é que o estudo da metamemória no envelhecimento supõe que pontuações em questionários como o MIA deveriam estar positivamente correlacionadas com desempenho em tarefas de memória. Isto é, indivíduos com pontuação elevada em questionários de meta-memória deveriam ter melhor desempenho de memória do que indivíduos com pontuação baixa. Entretanto, a literatura internacional documenta que a correlação entre 
a meta-memória e o desempenho objetivo de memória é moderada e está ao redor de 0,30 (Cavanaugh \& Green, 1990; Dixon, Hultsch, \& Hertzog, 1988; Lovelace, 1990), sugerindo que talvez a relação mais forte seja entre a meta-memória e as variáveis mediadoras, como esforço, motivação e perseverança.

\section{Auto-eficácia}

De acordo com Bandura (1977, 1981, 1986, 1997), o conceito da auto-eficácia refere-se à avaliação que um individuo faz de sua habilidade de realizar uma tarefa dentro de um certo domínio. A teoria da auto-eficácia prevê que o nível de confiança do individuo em suas habilidade é um forte motivador e regulador de seus comportamentos. Bandura defende que o indivíduo que se percebe capaz de realizar uma determinada tarefa, faz maior esforço para realizá-la, tem maior motivação para concluí-la e persevera mais tempo na sua realização do que o indivíduo com baixa auto-eficácia. Em um estudo meta-analítico envolvendo 36 estudos sobre auto-eficácia e desempenho acadêmico, Multon, Brown e Lent (1991) documentaram que 14\% da variância nos testes de desempenho era explicada pela auto-eficácia.

Bandura (1977, 1981, 1986, 1997) propõe também que existam pelo menos quatro fontes possíveis para a auto-eficácia. A fonte mais importante seria o próprio desempenho da pessoa nas tarefas em um determinado domínio. Por exemplo, uma criança que freqüentemente marca diversos gols em suas aulas de futebol, provavelmente irá incorporar este bom desempenho em sua auto-avaliação como jogador, e terá uma auto-eficácia elevada neste domínio. A auto-eficácia também pode ser influenciada pela observação do desempenho de outras pessoas - que pode nos levar a concluir que faríamos melhor ou pior do que os outros fazem. As outras fontes da auto-eficácia seriam a persuasão verbal de outras pessoas, que podem nos convencer que somos ou não capazes de realizar algo. Finalmente, a percepção de nossos estados fisiológicos também pode afetar nossa auto-eficácia, pois se nos sentimos ansiosos, amedrontados frente a certas tarefas, podemos inferir que nos sentimos assim porque não somos capazes de realizá-las.

O conceito de auto-eficácia é importante para o estudo do envelhecimento da memória porque a baixa auto-eficácia pode ajudar a explicar os déficits na memória do idoso. Diversos autores (Berry \& West, 1994; Cavanaugh, 1996; Welch \& West, 1995) afirmam que os idosos são mais vulneráveis a auto-eficácia rebaixada, pois vivenciam dificuldades cognitivas com maior freqüência e são fortemente afetados pelos estereótipos negativos sobre o envelhecimento. As falhas na memória dos idosos são mais notadas e podem ser interpretadas como sinais de um declínio irreversível. Este processo pode levar à insegurança, que, por sua vez, pode afetar o desempenho cognitivo, fechando um círculo vicioso (West, Welch \& Yassuda, 2000). É plausível também que idosos com senso de auto-eficácia rebaixada passem a evitar tarefas que envolvam memorização, com implicações negativas em seu cotidiano. Evidências obtidas em pesquisas internacionais sugerem que a auto-eficácia dos idosos para memória é significativamente mais baixa que a dos adultos jovens, e que a autoeficácia tende a se correlacionar positivamente com o desempenho objetivo em tarefas de memorização (Berry, West \& Dennehey, 1989; West \& Berry, 1994).

A literatura científica a respeito do envelhecimento cognitivo contém diversas medidas de avaliação da auto-eficácia para a memória. Alguns pesquisadores utilizaram como medida da auto-eficácia uma única pergunta, como por exemplo, uma previsão de desempenho pelo participante frente a uma tarefa de memória: "Quantas palavras você vai conseguir lembrar na ordem correta de uma lista com 12 palavras?” (Rebok \& Balcerak, 1989).

Outros pesquisadores desenvolveram escalas para avaliar a auto-eficácia, como o Memory Self-Efficacy Questionnaire (MSEQ) elaborado por Berry e colaboradores (1989). O MSEQ é baseado na metodologia de Bandura para a avaliação da auto-eficácia, pois os participantes devem avaliar quão bem poderiam realizar uma tarefa de memória dentro de cinco graus de complexidade. Por exemplo, para uma tarefa de memorização de palavras o participante responde se seria capaz de memorizar 12 palavras, 10 palavras, 8 palavras, 6 palavras ou 4 palavras. Esta avaliação constitui o nível da auto-eficácia do indivíduo para uma determinada tarefa. A cada grau de dificuldade a pessoa também deve expressar seu grau de confiança se conseguiria lembrar determinado número de palavras, o que constitui a variável força da auto-eficácia. O MSEQ na sua versão original inclui 10 subescalas, isto é, o participante avalia sua capacidade de realizar 10 tarefas de memória, apresentadas em graus hierárquicos de dificuldade. Versões atuais do questionário incluem 4 ou 6 subescalas, pois estudos posteriores evidenciaram a manutenção de suas características psicométricas em versões reduzidas (West \& Berry, 1994; West, Welch \& Thorn, 2001). Desde sua elaboração, o MSEQ tem se mostrado um instrumento útil para a avaliação da auto-eficácia para memória em populações idosas, em particular, em estudos de treino de memória. Nos estudos de treino o MSEQ tem sido utilizado para avaliar possíveis alterações na auto-eficácia em função da aquisição de estratégias mnemônicas (West \& cols., 2000).

Em resumo, o estudo do envelhecimento cognitivo humano vem progredindo rapidamente. Sabe-se hoje com bastante certeza que certos déficits de memória fazem parte do envelhecimento saudável. Entretanto, permanece aberta a discussão a respeito dos fatores motivadores de tal declínio. Uma das hipóteses em investigação é a importância das percepções e crenças dos idosos a respeito da memória. Os dados revisados acima sugerem uma relação importante entre os conceitos de meta-memória e autoeficácia e a memória do idoso.

Entretanto, deve-se ressaltar que os estudos mencionados acima foram realizados em países desenvolvidos com idosos com pelo menos oito anos de escolaridade. O estudo do envelhecimento saudável através da perspectiva da psicologia cognitiva no Brasil encontra-se em sua infância. Em primeiro lugar, existe a necessidade de verificarmos se os resultados que são bem aceitos na literatura internacional são também válidos para a população brasileira. Por exemplo, necessitamos saber se o declínio da memória causado pelo envelhecimento na nossa população é da mesma magnitude do declínio observado em 
outros países. Necessitamos também investigar se a relação documentada entre a meta-memória e o desempenho objetivo também se mantém junto à nossa população idosa. Para tal fim, estudos brasileiros precisam validar instrumentos de pesquisa necessários para o avanço deste campo no Brasil.

$\mathrm{O}$ estudo descrito a seguir teve como objetivo avaliar a validade da versão em português dos questionários MIA, MSEQ, de quatro listas de supermercado e quatro histórias curtas como medidas de desempenho de memória, junto a uma amostra da população brasileira.

\section{Método}

\section{Participantes}

Participaram deste estudo de validação 33 jovens universitários e 27 idosos membros de duas organizações para a terceira idade. Os jovens tinham em média 27,2 anos $(d p=10,1)$ e 12,3 anos de escolaridade $(d p=2,1)$, enquanto os idosos tinham em média 62,6 $\operatorname{anos}(d p=6,7)$ e 10,4 anos de escolaridade ( $d p=3,61)$. Quanto à saúde, os participantes escolheram um número entre 1 e 10 (1 igual a saúde excelente e 10 igual a saúde ruim) para representar como avaliavam sua saúde física. Em média, os jovens classificaram sua saúde como $2,5(d p=1,6)$, e os idosos como 3,0 $(d p=2,0)$, revelando que ambos os grupos consideravam boa sua saúde. Testes estatísticos de MannWhitney (Conover, 1971) compararam os dois grupos quanto à escolaridade e à saúde e revelaram que jovens e idosos eram equivalentes quanto à saúde, mas que a diferença a favor dos jovens quanto à escolaridade era significativa $(p=0,035)$. Trinta jovens eram do sexo feminino e 3 do sexo masculino, e 23 idosos eram do sexo feminino e 4 eram do sexo masculino.

Os participantes jovens foram recrutados em uma universidade particular no estado de São Paulo, e eram alunos do $1^{\circ}$ ano do curso de psicologia. Os idosos foram recrutados em duas organizações que promovem o bem-estar físico e social de pessoas em terceira idade, também no estado de São Paulo.

\section{Materiais}

Questionários de meta-memória e auto-eficácia: O questionário de metamemória MIA e o questionário de auto-eficácia MSEQ foram traduzidos do original em inglês para o português pela primeira autora, que é fluente nas duas línguas. Posteriormente, a versão em português dos questionários foi submetida à retrotradução para o inglês por uma tradutora profissional, que não teve acesso ao questionário original. A primeira página dos questionários no original, dos questionários em Português e da retrotradução está no Anexo A, e pode-se observar que a retrotradução e os questionários originais são fiéis um ao outro. Os questionários não foram apresentados na íntegra devido a limitações de espaço, mas podem ser obtidos junto à primeira autora. Neste estudo, a versão do MSEQ inclui seis tarefas diferentes de memorização: listas de supermercado, histórias curtas, nomes de pessoas, localização de objetos, tarefas a serem realizadas e listas de palavras.
Os questionários no português foram também submetidos à avaliação de juízes especialistas, duas gerontólogas e um geriatra, todos pesquisadores com interesse em memória e envelhecimento. Os três juízes demonstraram através de suas avaliações que as questões eram pertinentes ao objetivo de cada questionário, e um deles fez algumas sugestões para o aperfeiçoamento do português no questionário MIA, que foram incorporadas ao mesmo.

Medidas de desempenho de memória: As listas de supermercado originais foram elaboradas por West e Thorn (2001), seguindo o procedimento descrito a seguir: 1) 4 pesquisadores identificaram um grande número de itens de supermercado e categorias bem definidas que pudessem organizá-los; 2) 9 juízes identificaram a melhor categoria para cada item e cada item foi julgado como um exemplar de freqüência baixa, moderada ou alta em sua categoria; 3) somente itens julgados como sendo de alta freqüência por pelo menos sete juizes foram incluídos nas listas finais; 4) cada lista foi construída com 8 categorias distintas com 5 itens em cada, formando um total de 40 itens por lista.

Para a versão brasileira, as listas foram traduzidas pela primeira autora, e os itens não encontrados com freqüência em nossos supermercados foram substituídos por itens mais comuns. $\mathrm{Na}$ versão brasileira das listas, uma categoria contendo 5 itens foi eliminada de cada lista. Assim, as listas brasileiras contêm 35 itens. Utilizou-se como critério a eliminação da categoria menos comum em supermercados brasileiros. Originalmente, nos EUA as listas foram elaboradas com muitos itens, pois foram utilizadas em estudos envolvendo jovens e idosos, e os jovens americanos lembravam de quase todos os itens quando a lista era menor. Considerando a escolaridade mais baixa do idoso brasileiro, julgamos que listas de 40 itens seriam demasiadamente difíceis e decidimos utilizar listas com 35 itens.

A primeira versão em português das quatro listas foi testada em um estudo piloto que incluiu 40 jovens universitários e 36 idosos saudáveis. A análise dos dados deste estudo revelou que duas listas eram mais difíceis e geravam desempenho mais baixo. Estas listas continham itens que nunca eram recordados pelos participantes. Estes itens eram artigos de escritório e de papelaria, que não são comprados com muita freqüência em supermercados brasileiros. Essas categorias, uma em cada lista considerada difícil, foram substituídas por duas novas categorias bastante comuns em nossos supermercados: legumes e verduras. Esta segunda versão das quatro listas foi utilizada neste estudo. No Anexo B reproduzimos uma das listas como ilustração. As outras três podem ser obtidas junto à primeira autora.

As 4 histórias foram selecionadas do manual desenvolvido por Dixon, Hultsch e Hertzog (1989) - Manual of twenty-five three tiered structurally equivalent text for use in aging research. As histórias foram traduzidas e adaptadas ao contexto brasileiro, quando necessário, pela primeira autora. As histórias originais são equivalentes quanto à estrutura semântica, com um número comparável de proposições. A tradução e adaptação tentaram manter estas características inalteradas o máximo possível, mantendo 63 proposições em cada história. Consideramos como uma proposição uma unidade mínima, que possuísse significado e pudesse ser recordada de maneira 
independente. No Anexo C reproduzimos uma das histórias como ilustração. As outras três podem ser obtidas junto à primeira autora.

As 4 listas e as 4 histórias também foram submetidas à avaliação dos juízes especialistas. Pedimos para os juízes avaliarem: as categorias de cada lista de supermercado (pedimos que categorizassem as 4 listas para confirmarmos as categorias esperadas), a popularidade dos itens das listas em supermercados brasileiros (pedimos para os juízes circularem itens não comuns), a clareza lingüística das quatro histórias e sua equivalência em termos de conteúdo emocional e grau de dificuldade para recordação.

Em geral, os juízes avaliaram as listas e histórias positivamente quanto à adequação das mesmas aos objetivos da pesquisa. Especificamente, os juízes sugeriram que alguns itens presentes nas listas de supermercado não são comuns em supermercados brasileiros e sugeriram algumas trocas. Um juiz também sugeriu a troca de algumas palavras em uma das histórias. Estas sugestões serão implementadas nas futuras versões das listas e histórias (maiores detalhes sobre a avaliação dos especialistas podem ser obtidos junto à primeira autora).

\section{Procedimentos}

Os participantes foram convidados a participar de uma pesquisa sobre memória. A coleta de dados foi realizada em grupos de aproximadamente 15 pessoas. Todos preencheram e assinaram Termo de Consentimento Livre e Esclarecido. A seguir, receberam os protocolos contendo o questionário sóciodemográfico e os questionários MIA e MSEQ. O pesquisador leu as instruções em voz alta e pediu aos participantes que preenchessem os questionários. O preenchimento dos questionários durou cerca de 50 minutos. Durante este período o pesquisador percorreu a sala tirando dúvidas. Quando todos haviam terminado, os protocolos foram entregues e conferidos, e logo após, as duas tarefas de memorização foram realizadas. Os participantes tiveram 3 minutos para estudar a história e a seguir
5 minutos para escrever o que se lembravam do que haviam lido. Posteriormente, receberam uma lista de supermercado com 35 itens e puderam estudá-la por 3 minutos. Em seguida tiveram mais 3 minutos para escrever todos os itens que conseguissem lembrar. Durante cada sessão um número equivalente de cada versão das histórias e das listas foi distribuído entre os participantes.

Dezoito jovens do grupo original preencheram os questionários MIA e MSEQ pela segunda vez 3 meses mais tarde para que a fidedignidade dos questionários pudesse ser calculada. Estes questionários foram preenchidos em casa, e entregues na semana seguinte.

\section{Resultados}

O nível de significância adotado para os testes estatísticos foi de $5 \%$, ou seja, $p<0,05$.

\section{MIA}

Como se pode observar no Anexo A, o MIA é composto por 108 questões que se subdividem em sete sub-escalas de acordo com a Tabela 1.

Para analisar a consistência interna do MIA foi calculado o coeficiente $\alpha$ de Cronbach (Carmines \& Zeller, 1979; Cronbach, 1951), para o questionário todo e para cada uma das sete subescalas. Valores de $\alpha$ acima de 0,80 indicam alta consistência interna, embora valores acima de 0,60 já indiquem consistência interna adequada. Os resultados indicaram alta consistência interna para o instrumento como um todo $(\alpha=0,79)$, e para todas as sub-escalas, com exceção da sub-escala Controle, que apresentou consistência intermediária (ver Tabela 2). Os resultados sugerem características psicométricas muito similares às características do questionário original (ver Dixon \& cols., 1988), com consistência interna elevada no total do questionário e intermediária para a sub-escala Controle (Locus no original).

Tabela 1

Estrutura de Sub-escalas do MLA

\begin{tabular}{lc}
\hline Sub-escala & \multicolumn{1}{c}{ Número do item } \\
\hline Estratégia & $3,6,11,17,25,29,36,48,57,60,64,67,75$, \\
$(+=$ maior utilização) & $81,85,94,98,108$ \\
Tarefa & $1 r, 20 r, 21 r, 31 r, 34 r, 43 r, 44 r, 50 r, 51 r, 72,74 r$, \\
$(+=$ maior conhecimento) & $80 r, 90 r, 93 r, 96 r, 107$ \\
Capacidade & $2 r, 9 r, 19 r, 27,49 r, 52 r, 59,62 r, 71 r, 77 r, 88 r$, \\
$(+=$ maior capacidade) & $91 r, 95 r, 97 r, 100 r, 104 r, 105 r$ \\
Mudança & $10 r, 14,16,18 r, 28,30,32,38,39,41,45 r, 54$, \\
$(+=$ maior estabilidade) & $56,58,76 r, 82 r, 89,92 r$ \\
Ansiedade & $5 r, 8 r, 12 r, 15 r, 22 r, 23 r, 42 r, 53 r, 66 r, 70 r, 78 r$, \\
(+= maior percepção) & $84 r, 87,99 r$ \\
Metas & $4 r, 7 r, 13 r, 24,26,37 r, 40 r, 46 r, 47 r, 55 r, 65 r$, \\
(+= melhor desempenho) & $68 r, 79 r, 83 r, 86 r, 103 r$ \\
Controle & $33,35,61 r, 63 r, 69 r, 73,101,102 r, 106 r$ \\
(+= maior percepção de controle) & \\
\hline Nota. Cada item é corrigido de acordo com uma escala Likert de 5 pontos. Os itens marcados com “r” são \\
corrigidos em reverso, isto é, 1=5, 5=1, 2=4, 4=2.
\end{tabular}


Tabela 2

Coeficientes de Consistência Interna do MLA

\begin{tabular}{ll}
\hline Sub-escalas & $\alpha$ de Cronbach \\
\hline Total & 0,79 \\
Estratégia & 0,86 \\
Tarefa & 0,79 \\
Capacidade & 0,81 \\
Mudança & 0,87 \\
Ansiedade & 0,85 \\
Metas & 0,80 \\
Controle & 0,66 \\
\hline Nota. $n=60$ &
\end{tabular}

Para comparar os escores de cada sub-escala entre os grupos etários foi utilizado o teste de Mann-Whitney (Conover, 1971), que compara a soma dos postos (ou ranks) das observações entre os grupos. Para a sub-escala Tarefa e Mudança foram encontradas diferenças estatisticamente significativas entre jovens e idosos (ver Tabela 3), indicando que estas são as sub-escalas mais sensíveis às diferentes percepções sobre a memória entre jovens e idosos. A pontuação mais elevada entre os idosos na sub-escala Tarefa sugere que os idosos da amostra tinham maior conhecimento sobre os processos básicos da memória do que os jovens. Em contrapartida, a pontuação mais elevada dos jovens na sub-escala Mudança sugere que estes não relatam uma percepção de declínio na sua habilidade de memória.

Quando os resultados das sub-escalas Estratégia, Tarefa e Metas foram agrupados para formar o fator Conhecimento, e as sub-escalas Capacidade, Controle e Mudança foram somadas para formar o fator Auto-Eficácia, como sugeriram as análises fatoriais realizadas com o questionário (Hertzog \& cols., 1987), as diferenças encontradas entre os grupos etários foram significativas para os dois fatores. A literatura internacional sugere que o fator Auto-eficácia é mais sensível ao envelhecimento, do que o fator Conhecimento para o qual não são esperadas diferenças entre os dois grupos. Para a amostra estuda foram encontradas diferenças para os dois fatores, sugerindo que os idosos teriam maior conhecimento sobre o funcionamento básico da memória, enquanto os jovens teriam percepções e sentimentos mais positivos frente à memória. A sub-escala Ansiedade também revelou diferenças significativas entre jovens e idosos, sugerindo que os idosos se sentem mais ansiosos frente aos desafios de memória (ver Tabela 3).

\section{MSEQ}

O questionário MSEQ gera duas variáveis de interesse: nível da auto-eficácia, e a força da auto-eficácia. O nível corresponde ao número de "sim" que o participante circula a cada sub-escala (cada tarefa de memória). A força corresponde à média das porcentagens assinaladas em cada sub-escala. Foram analisados o Nível da AutoEficácia da Memória (NAE) e a Força da Auto-Eficácia da Memória (FAE) para as seis tarefas de memorização apresentadas no questionário: Lista de Supermercado (L), História (H), Localização de Objetos (O), Nomes de Pessoas (N), Lista de Coisas a Fazer (C) e Lista de Palavras (P), ver Anexo A.

A Tabela 4 apresenta os coeficientes $\alpha$ de Cronbach para o MSEQ, considerando dois domínios (Nível e Força), cada um composto por seis itens (L, H, O, N, C e P). Verifica-se alta consistência interna para o instrumento como um todo $(a=0,89)$ e para os dois domínios em separado.

Tabela 4

Coeficientes de Consistência Interna do MSEQ

\begin{tabular}{ll}
\hline Sub-escalas & $\alpha$ de Cronbach \\
\hline Total & 0,89 \\
Nível (L,H,O,N,C,P) & 0,81 \\
Força (L,H,O,N,C,P) & 0,81 \\
\hline Nota $n=58$ &
\end{tabular}

A Tabela 5 apresenta as comparações dos escores de cada subescala do MSEQ entre os grupos etários. Pode-se observar que para todas as sub-escalas o nível e a força da auto-eficácia são mais altos para os jovens do que para os idosos, confirmando a literatura internacional (Berry \& cols., 1989). Entretanto, estas diferenças somente atingem níveis estatisticamente significativos para a tarefa de memorização de nomes, para a qual os jovens reportam níveis bem mais altos de auto-eficácia do que os idosos. Quando se calcula uma média geral do nível e da força da auto-eficácia para as seis subescalas, nota-se que a diferença entre jovens e idosos aproxima-se da significância estatística para a variável nível da auto-eficácia.

Tabela 3

Comparação entre Jovens e Idosos para as Sub-escalas do MLA

\begin{tabular}{lllll} 
& \multicolumn{2}{c}{ Jovens } & $(n=27)$ & \multicolumn{2}{c}{ Idosos $(n=33)$} \\
\hline Sub-escalas & $m$ & $d p$ & $m$ & $d p$ \\
Estratégia & 3,36 & 0,66 & 3,65 & 0,55 \\
Tarefa $^{a}$ & 3,68 & 0,42 & 4,01 & 0,41 \\
Capacidade $_{\text {Mudança }}$ & 3,26 & 0,51 & 3,05 & 0,55 \\
Ansiedade $^{\mathrm{b}}$ & 3,14 & 0,54 & 2,54 & 0,56 \\
Metas & 3,18 & 0,61 & 3,60 & 0,73 \\
Controle $_{\text {Fator conhecimento }}$ & 3,89 & 0,50 & 4,06 & 0,38 \\
Fator auto-eficácia $^{\mathrm{a}}$ & 3,82 & 0,61 & 3,63 & 0,42 \\
\hline
\end{tabular}

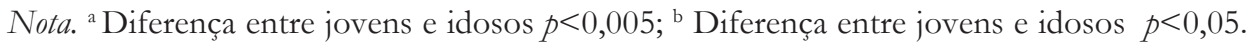


Tabela 5

Comparação entre Jovens e Idosos para as Sub-escalas do MSEQ

\begin{tabular}{|c|c|c|c|c|c|c|}
\hline \multirow[b]{2}{*}{$\underline{\text { Sub-escalas }}$} & \multicolumn{3}{|c|}{ Jovens } & \multicolumn{3}{|c|}{ Idosos } \\
\hline & $m$ & $d p$ & $n$ & $m$ & $d p$ & $n$ \\
\hline \multicolumn{7}{|l|}{ Nível } \\
\hline Lista & 3,34 & 1,26 & 32 & 3,15 & 1,29 & 27 \\
\hline História & 3,72 & 1,08 & 32 & 3,37 & 1,42 & 27 \\
\hline Objetos & 3,56 & 1,22 & 32 & 3,37 & 1,18 & 27 \\
\hline Nomes a & 3,75 & 1,16 & 32 & 3,00 & 1,27 & 27 \\
\hline Coisas a fazer ${ }^{\mathrm{b}}$ & 3,72 & 1,11 & 32 & 3,15 & 1,19 & 26 \\
\hline Palavras ${ }^{\mathrm{b}}$ & 3,09 & 1,06 & 32 & 2,62 & 1,02 & 26 \\
\hline Nível (média 6 sub-escalas) ${ }^{\mathrm{b}}$ & 3,53 & 0,79 & 32 & 3,07 & 0,88 & 26 \\
\hline \multicolumn{7}{|l|}{ Força } \\
\hline Lista & 52,32 & 20,26 & 31 & 51,78 & 22,41 & 27 \\
\hline História & 51,35 & 16,17 & 31 & 50,81 & 22,60 & 27 \\
\hline Objetos & 53,10 & 24,26 & 31 & 51,63 & 21,80 & 27 \\
\hline Nomes b & 55,10 & 24,73 & 31 & 43,70 & 20,60 & 27 \\
\hline Coisas a fazer & 56,97 & 20,41 & 31 & 50,92 & 21,14 & 26 \\
\hline Palavras & 42,58 & 15,97 & 31 & 41,08 & 17,63 & 26 \\
\hline Força (média 6 sub-escalas) & 51,90 & 14,06 & 31 & 47,69 & 1544 & 26 \\
\hline
\end{tabular}

Nota. ${ }^{a}$ Diferença entre jovens e idosos $p<0,05 ;{ }^{b}$ Diferença entre jovens e idosos $p<0,1$.

\section{Estabilidade Temporal do MIA e do MSEQ}

Para analisar a correlação entre os momentos de teste e re-teste (3 meses após) do MIA e do MSEQ foi utilizado o coeficiente de correlação de Spearman (Conover, 1971). As correlações (ver Tabela 6) revelam que o questionário MIA apresenta certa estabilidade

Tabela 6

Correlações entre Teste e Re-teste para o MIA e o MSEQ por Sub-escalas Instrumento/sub-escala $\mathrm{r}$

MIA

$\begin{array}{lr}\text { Estratégia }^{\text {a }} & 0,83 \\ \text { Tarefa }^{a} & 0,57 \\ \text { Capacidade }^{\text {a }} & 0,70 \\ \text { Mudança }^{\text {a }} & 0,57 \\ \text { Ansiedade }^{\text {a }} & 0,77 \\ \text { Metas }^{\text {a }} & 0,66 \\ \text { Controle }^{\mathrm{b}} & 0,43 \\ \text { Fator conhecimento }^{\text {a }} & 0,67 \\ \text { Fator auto-eficácia }^{a} & 0,48\end{array}$

MSEQ

Nível

\begin{tabular}{|c|c|}
\hline Lista & 0,17 \\
\hline História $^{a}$ & 0,67 \\
\hline Objetos & 0,29 \\
\hline Nomes a & 0,62 \\
\hline Coisas a fazer & 0,47 \\
\hline Palavras & 0,37 \\
\hline média 6 sub-escalas) ${ }^{a}$ & 0,56 \\
\hline Lista & 0,23 \\
\hline História $^{a}$ & 0,55 \\
\hline Objetos ${ }^{a}$ & 0,56 \\
\hline Nomes & 0,27 \\
\hline Coisas a fazer ${ }^{\mathrm{a}}$ & 0,62 \\
\hline Palavras & 0,38 \\
\hline média 6 sub-escalas) ${ }^{a}$ & 0,58 \\
\hline
\end{tabular}

$\frac{\text { Força (média } 6 \text { sub-escalas) }}{\text { Nota. } \mathrm{N}=18 . \quad{ }^{\mathrm{a}} p<0,05^{\mathrm{b}} p<0,1}$ temporal; para seis sub-escalas a correlação entre os dois momentos é significativa, entretanto, algumas são de magnitude modesta. A correlação para a sub-escala Controle se aproxima de níveis significativos.

A escala MSEQ mostrou-se relativamente estável entre os dois momentos de aplicação. A correlação para algumas de suas subescalas não atingiu significância estatística, entretanto, quando os escores das seis sub-escalas são condensados, encontra-se correlações moderadas entre o teste e o re-teste da escala MSEQ, sugerindo certo grau de estabilidade na auto-eficácia dos participantes jovens que refizeram os questionários (ver Tabela 6).

\section{Medidas de Desempenho de Memória}

Quanto às medidas de desempenho de memória, as quatro versões das listas de supermercado e as quatro pequenas histórias foram comparadas entre si através do teste de Kruskal-Wallis. Como demonstra a Tabela 7, as histórias B, C e D geraram escores estatisticamente equivalentes quando os dois grupos etários estão juntos na análise, enquanto a história A tende a gerar escores mais baixos, apresentando-se mais difícil que as outras histórias. Análise do conteúdo desta história revela que esta contém mais informações numéricas do que as outras (ver Anexo C), apesar de todas as histórias conterem 63 proposições. A próxima versão da lista A deverá incluir pequenas mudanças para eliminar esta disparidade.

Tabela 7

Comparação entre as Versões Alternativas das Histórias e Listas

\begin{tabular}{lllllll}
\hline Versão & \multicolumn{3}{c}{ Histórias } & \multicolumn{3}{c}{ Listas } \\
\hline & $m$ & $d p$ & $n$ & $m$ & $d p$ & $n$ \\
\hline A & $19,31^{\text {a }}$ & 6,93 & 13 & 14,07 & 4,63 & 14 \\
B & 25,07 & 11,38 & 15 & 13,25 & 5,22 & 12 \\
C & 27,33 & 6,55 & 15 & 13,79 & 3,60 & 14 \\
D & 30,18 & 8,13 & 11 & 13,79 & 3,87 & 14 \\
\hline
\end{tabular}

Nota. Teste de Kruskal-Wallis: histórias $p<0,05$, versão A diferente das demais histórias, listas $p>0,05$, listas equivalentes. 
A análise das listas de supermercado revelou que as quatro versões são estatisticamente equivalentes em seu grau de dificuldade, pois geram escores comparáveis (ver Tabela 7).

O desempenho nas tarefas de memorização entre os dois grupos etários foi comparado através do teste de Mann-Whitney. Como seria esperado, os jovens tiveram desempenho superior ao dos idosos nestas tarefas de memória episódica (ver Tabela 8).

Tabela 8

Desempenho de Jovens e Idosos nas Tarefas de Memória

\begin{tabular}{lllllll}
\hline Grupo & \multicolumn{3}{l}{ História } & \multicolumn{4}{c}{ Lista } \\
\hline & $m$ & $d p$ & $n$ & $m$ & $d p$ & $n$ \\
Jovens & 27,89 & 8,99 & 27 & 15,00 & 4,36 & 27 \\
Idosos & 22,81 & 8,76 & 27 & 12,48 & 3,76 & 27 \\
\hline
\end{tabular}

Nota. Teste Mann-Whitney história $p<0,05$, lista $p<0,05$

\section{Correlações entre Medidas de Desempenho e de Meta- memória}

Finalmente, coeficientes de correlação de Spearman entre os questionários MIA e MSEQ e as tarefas de desempenho de memória para jovens e idosos incluídos na mesma análise revelaram uma correlação modesta, mas estatisticamente significante entre o desempenho nas histórias e o fator auto-eficácia do MIA. As correlações entre o desempenho nas listas e as medidas de metamemória não foram significativas. Estes resultados sugerem uma modesta relação entre auto-eficácia e desempenho objetivo em algumas tarefas de memória, como foi sugerido em artigos anteriores (Hertzog \& cols., 1988). É interessante notar também a modesta correlação entre as duas medidas de desempenho de memória, que sugere que um mesmo indivíduo pode ter desempenhos relativamente diferentes em tarefas que envolvem memorização.

Tabela 9

Correlação entre os Questionários e o Desempenho de Memória

\begin{tabular}{lccccc}
\hline & MIA-C & MIA-AE & NAE & FAE & Lista \\
\hline História & $-0,09$ & $0,33^{a}$ & 0,03 & 0,08 & $0,28^{a}$ \\
Lista & $-0,14$ & 0,19 & $-0,38$ & $-0,06$ & \\
\hline
\end{tabular}

Nota. ${ }^{a} p<0,05$

\section{Discussão}

A literatura cognitiva documenta através de inúmeros estudos que diferenças significativas são encontradas quando comparamos o desempenho de jovens e idosos em tarefas de memória episódica. Uma das possíveis explicações em investigação para o desempenho inferior dos idosos é que talvez existam diferenças importantes na meta-memória de jovens e idosos. Investiga-se se percepções, crenças e afetos negativos sobre a memória, freqüentemente mais comuns entre idosos, têm impacto negativo sobre o processo de memorização.

O presente estudo representa um esforço de avançar esta área de investigação junto à população brasileira. Foram traduzidos e adaptados para o português um questionário de meta-memória (MIA) e um questionário de auto-eficácia (MSEQ), assim como quatro histórias curtas e quatro listas de supermercado, como instrumentos de avaliação da memória episódica. Foi realizado também um estudo preliminar de validação desses instrumentos. Ao avaliarmos os resultados deste estudo preliminar devemos considerar o tamanho limitado da amostra. Futuros estudos com maior número de participantes serão necessários para que os estes resultados possam ser confirmados.

Os resultados revelaram que a versão em português do MIA e do MSEQ podem ser bons instrumentos de pesquisa empírica junto à população brasileira. A retrotradução indica que a versão em português é lingüisticamente fiel aos questionários originais, sendo sua adequação também confirmada pela avaliação dos juízes especialistas. A avaliação das características psicométricas dos questionários revelou que os mesmos apresentam boa consistência interna e certo grau de fidedignidade, pois apresentaram-se relativamente estáveis na avaliação do teste e reteste em uma parcela da amostra três meses após a avaliação inicial.

Na literatura internacional, o MIA e o MSEQ tradicionalmente geram diferenças significativas entre jovens e idosos. Esta característica também foi encontrada junto à nossa população. No MIA, os idosos revelaram maior conhecimento ou consciência dos processos de memorização, maior percepção de mudança nas habilidades de memória com o passar do tempo e maior ansiedade frente a situações que envolvam a memória. No MSEQ, os idosos revelaram auto-eficácia mais baixa quando comparados aos jovens, entretanto, as diferenças não foram estatisticamente significantes. Para a variável nível de auto-eficácia, a diferença entre os grupos se aproximou da significância estatística. É provável que em amostras maiores a diferença entre os grupos etários atinja significância.

As quatro versões dos instrumentos de desempenho também geraram escores diferentes entre jovens e idosos, com os jovens apresentando desempenho superior na memorização das listas e das histórias como era esperado. Quatro versões equivalentes de historias e listas foram avaliadas, pois versões alternativas são de extremo valor em estudos que envolvem múltiplos retestes, como é o caso de estudos de treino de memória. As quatro listas geraram escores equivalentes, mostrando-se do mesmo grau de dificuldade. Uma das histórias gerou escores mais baixos que as outras três, mostrando-se mais difícil, provavelmente devido a maior presença de informações numéricas. Esta lista será alterada, mantendo o mesmo número de proposições, mas algumas das informações numéricas serão substituídas por outro tipo de informação.

É interessante notarmos a ausência de correlações significativas entre os questionários MIA e MSEQ. Em termos teóricos, a autoeficácia faz parte do conceito de meta-memória, como visto anteriormente, então, poder-se-ia supor que o fator Auto-eficácia do questionário MIA estivesse positivamente correlacionado com as variáveis derivadas do questionário MSEQ. Entretanto, esta correlação não foi encontrada na amostra estudada. Novamente, devemos ressaltar que o poder estatístico das análises foi limitado pelo número restrito de participantes. Entretanto, uma outra explicação para a ausência desta correlação é que cada questionário avalia a auto-eficácia operacionalmente de maneira distinta. No MIA, por exemplo, o participante deve julgar o quanto concorda com a frase genérica "Sou bom para lembrar nomes", enquanto no MSEQ ele deve julgar 
objetivamente se conseguiria ou não memorizar os nomes de 10 pessoas após terem sido apresentadas em fotos. É possível que o MIA esteja avaliando uma percepção generalizada de desempenho, enquanto o MSEQ esteja mais próximo de uma previsão de desempenho.

Em harmonia com a literatura internacional, os resultados revelaram uma modesta, mas significativa correlação entre os escores na memorização de histórias e o fator auto-eficácia do MIA, encontrada também nos estudos de validação do questionário original (Dixon \& Hultsch, 1983a, 1983b). De acordo com os autores do questionário, as modestas correlações que geralmente são encontradas entre medidas de desempenho e as sub-escalas do MIA não indicam falta de validade de construto do MIA, pois este inclui muitas perguntas sobre memória que não são passíveis de verificação concreta no cotidiano dos participantes. O MIA é na verdade um instrumento de avaliação de conhecimentos, crenças e afetos sobre a memória que pode ser de grande valor para pesquisas sobre o envelhecimento normal e patológico da memória.

Espera-se que a adaptação do MIA e do MSEQ estimule outras pesquisas sobre o conceito de meta-memória e sobre a teoria da auto-eficácia, assim como a utilidade destes conceitos para o estudo da memória no envelhecimento. Os resultados do presente estudo sugerem que os instrumentos de pesquisa apresentados são promissores. Futuras pesquisas deveriam replicar estes resultados com amostras maiores, e continuar a investigação sobre a trajetória da meta-memória e da auto-eficácia ao longo do desenvolvimento. Estudos de treino de memória para idosos também podem se valer dos questionários e das medidas de desempenho para avaliar a eficácia das intervenções.

\section{Referências}

Abranson, L. Y., Seligman, M. E. \& Teasdale, J. D. (1978). Learned helpness: Critique and reformulation. Journal of Abnormal Psychology, 87, 49-74.

Abreu, V. P. S. \& Xavier, G. F. (2001). Memória e envelhecimento. Insight, junho, 11-16. Bandura, A. (1977). Self-efficacy: Toward a unifying theory of behavioral change. Psychological Revien, 85, 191-215.

Bandura, A. (1981). Self-referent thought: A developmental analysis of self-efficacy. Em J. H. Flavell \& L. Ross (Orgs.), Social and cognitive development: Frontiers and possible futures (pp. 200-239). Cambridge: Cambridge University Press.

Bandura, A. (1986). Social foundations of thought and action: A social cognitive theory. Englewood Cliffs, NJ: Prentice Hall.

Bandura, A. (1997). Self-efficacy: The exercise of control. New York: W.H. Freeman.

Berry, J. M. \& West, R. L. (1983). Cognitive self-efficacy in relation to personal mastery and goal setting across the life-span. International Journal of Behavioral Development, 16, 351-379.

Berry, J. M., West, R. L. \& Dennehey, D. M. (1989). Reliability and validity of the self-efficacy questionnaire. Developmental Psychology, 25, 701-713.

Carmines, E. G. \& Zeller, R. A. (1979). Reliability and validity assessment. New York: Sage.

Cavanaugh, J. C. (1996). Memory self-efficacy as a moderator of memory change. Em F. Blanchard-Fields \& T. M. Hess (Orgs.), Perspectives on cognitive change in adulthood and aging (pp. 488-507). New York: MacGraw-Hill.
Cavanaugh, J. C. \& Green, E.A. (1990). I believe, therefore I can: Self-efficacy beliefs in memory aging. Em E. A. Lovelace (Org.), Aging and cognition: Mental processes, self-awareness and interventions (pp. 189-230). Amsterdam: North-Holland.

Conover, W. J. (1971). Practical nonparametric statistics. New York: John Wiley \& Sons.

Cronbach, L. J. (1951). Coefficient alpha and the internal structure of tests. Psychometrika, 16, 297-334.

Dixon, R. A. \& Hultsch, D. F. (1983a). Metamemory and memory for text relationships in adulthood: A cross-validation study. Journal of Gerontology, 38, 689-694.

Dixon, R. A. \& Hultsch, D. F. (1983b). Structure and development of metamemory in adulthood. Journal of Gerontology, 38, 682-688.

Dixon, R. A., Hultsch, D. F. \& Hertzog, C. (1988). The metamemory in adulthood (MIA) questionnaire. Psychopharmacology Bulletin, 24, 671-688.

Dixon, R. A., Hultsch, D. F. \& Hertzog, C. (1989). A manual of twenty-five three-tiered structurally equivalent text for use in aging research. CRGCA Technical Report $\mathrm{n \#}$ 2, University of Victoria and Georgia Institute of Technology.

Flavell, J. H. (1971). First discussant's comments: What's memory development the development of? Human Development, 14, 272-278.

Flavell, J. H. \& Wellman, H. M. (1977). Metamemory. Em R. V. Kail \& J. W. Hagen (Orgs.), Perspectives on the development of memory and cognition (pp. 3-34). Hillsdale, NJ: Erlbaum.

Gilewski, M. J., Zelinski, E. M., Schaie, K. W. \& Thompson, L. W. (1983, agosto). Abbreviating the metamemory questionnaire: Factor structure norms for adults. Trabalho apresentado no encontro da American Psychological Association, Anaheim, CA.

Hertzog, C., Dixon, R. A., Schulenberg, J. E. \& Hultsch, D. F. (1987). On the differentiation of memory beliefs from memory knowledge: The factor structure of the metamemory in adulthood scale. Experimental Aging Research, 13, 101-107.

Hertzog, C., McGuire, C. L. \& Lineweaver, T. T. (1998). Aging, attributions, perceived control, and strategy use in a free recall task. Aging, Neuropsychology, and Cognition, 5, 85-106.

Hultsch, D. F., Hertzog, C., Dixon, R. A. \& Davidson, H. (1988). Memory selfknowledge and self- efficacy in the aged. Em M. L. Howe \& C. J. Brainerd (Orgs.), Cognitive development in adulthood: Progress in developmental research (pp. 65-92). New York: Springer.

Lachman, M. E. (1983). Perceptions of intellectual aging: Antecedent or consequence of intellectual functioning? Developmental Psychology, 19, 482-498.

Lovelace, E. A. (1990). Aging and metacognitions concerning memory function. Em E. A. Lovelace (Org.), Aging and cognition:Mental processes, self-awareness and interventions (pp. 152-188). Amsterdam: North-Holland.

Multon, K. D., Brown, S. D. \& Lent, R. W. (1991). Relation of efficacy beliefs to academic outcomes: A meta-analytic investigation. Journal of Counseling Psychology, 38, 30-38.

Rebok, G. W. \& Balcerak,L.J.(1989). Memory self-efficacy and performance differences in young and old adults: The effect of mnemonic training. Developmental Psychology, 25, 714-721.

Salthouse, T. A. (1991). Theoretical perspectives on cognitive aging. Hillsdale, NJ: Erlbaum.

Welch, D. W. \& West, R. W. (1995). Self-efficacy and mastery: Its applications to issues of environmental control, cognition and aging. Developmental Reviem, 15, 150-171.

West, R. L. \& Berry,J.M. (1994). Age declines in memory self-efficacy: General or limited to particular tasks and measures? Em J. D. Sinnott (Org.), Handbook of adult lifespan learning (pp. 426-446). New York: Greenwood Press.

West R. L. \& Thorn, R. M. (2001). Goal-setting, self-efficacy, and memory performance in older and younger adults. Experimental Aging Research, 27, 41-65.

West R. L., Welch, D. C. \& Thom, R. M. (2001). Effects of goal-setting and feedback on memory performance and beliefs among older and younger adults. Psychology and Aging, 16, 2, 240-250.

West, R. L., Welch, D. C. \& Yassuda, M. S. (2000). Innovative approaches to memory training for older adults. Em R. D. Hill, L. Backman, A. Stigsdotter-Neely (Orgs.), Cognitive rehabilitation in old age (pp. 81-105). Oxford, England: Oxford University Press.

Yassuda, M. S. (2002). Memória e envelhecimento saudável. Em E. V. Freitas, L. Py, A. L. Néri, F. A. X. Cançado, M. L. Gorzoni \& S. M. Rocha (Orgs.), Tratado de geriatria e gerontologia (pp. 914-920). Rio de Janeiro: Guanabara Koogan.

Recebido: 23/04/2003

$1^{a}$ revisão: 20/11/2003

Aceite final: $21 / 01 / 2004$

Sobre as autoras

Mônica Sanches Yassuda é pós-doutorada em Gerontologia na Universidade Estadual de Campinas, Mestre e Doutora em Psicologia do Desenvolvimento Humano pela Universidade da Flórida, Gainesville, EUA, professora doutora no curso de gerontologia da USP Leste.

Valéria Bellini Lasca é Mestre em Gerontologia pela Universidade Estadual de Campinas.

Anita Liberalesso Néri é Professora titular da Universidade Estadual de Campinas, professora e pesquisadora em Psicologia da Vida Adulta e Velhice. 


\section{Anexo A}

\section{MIA em Português exemplo $1^{\mathrm{a}}$. pagina}

1. Para a maioria das pessoas, fatos que são interessantes são mais fáceis de lembrar do que fatos que não são.
a. concordo totalmente
b. concordo
c. não sei
d. discordo
e. discordo totalmente

2. Eu sou boa/bom para lembrar nomes.
a. concordo totalmente
b. concordo
c. não sei
d. discordo
e. discordo totalmente

3. Você mantém uma lista ou anota datas importantes, como aniversários?
a. nunca
b. raramente
c. algumas vezes
d. freqüentemente
e. sempre

4. Ter uma boa memória é importante para mim.
a. concordo totalmente
b. concordo
c. não sei
d. discordo
e. discordo totalmente

5. Eu fico chateada(o), quando não consigo lembrar alguma coisa.
a. concordo totalmente
b. concordo
c. não sei
d. discordo
e. discordo totalmente

6. Quando você está procurando alguma coisa que guardou recentemente em um lugar errado, você tenta refazer seus passos para achar o objeto?
a. nunca
b. raramente
c. algumas vezes
d. freqüentemente
e. sempre

7. Eu acho que uma boa memória é motivo de orgulho.
a. concordo totalmente
b. concordo
c. não sei
d. discordo
e. discordo totalmente

8. Eu acho mais difícil lembrar das coisas quando estou chateada(o).
a. concordo totalmente
b. concordo
c. não sei
d. discordo
e. discordo totalmente

9. Eu sou bom/boa para lembrar de aniversários.
a. concordo totalmente
b. concordo
c. não sei
d. discordo
e. discordo totalmente

10. Eu consigo lembrar das coisas tão bem quanto no passado.
a. concordo totalmente
b. concordo
c. não sei
d. discordo
e. discordo totalmente

\section{MIA - Questionário Original 1ª página}

1. For most people, facts that are interesting are easier to remember than facts that are not.
a. agree strongly
b. agree
c. undecided
d. disagree
e. disagree strongly

2. I am good at remembering names.
a. agree strongly
b. agree
c. undecided
d. disagree
e. disagree strongly

3. Do you keep a list or otherwise note important dates such as birthdays and anniversaries?
a. never
b. rarely
c. sometimes
d. often
e. always

4. It is important to me to have a good memory.
a. agree strongly
b. agree
c. undecided
d. disagree
e. disagree strongly 
5. I get upset when I cannot remember something.
a. agree strongly
b. agree
c. undecided
d. disagree
e. disagree strongly

6. When you are looking for something you have recently misplaced, do you try to retrace your steps in order to locate it?
a. never
b. rarely
c. sometimes
d. often
e. always

7. I think a good memory is something of which to be proud.
a. agree strongly
b. agree
c. undecided
d. disagree
e. disagree strongly

8. I find it harder to remember things when I get upset.
a. agree strongly
b. agree
c. undecided
d. disagree
e. disagree strongly

9. I am good at remembering birthdates.
a. agree strongly
b. agree
c. undecided
d. disagree
e. disagree strongly

10. I can remember things as well as always.
a. agree strongly
b. agree
c. undecided
d. disagree
e. disagree strongly

\section{MIA- Retrotradução 1a. página}

1. For most people, interesting facts are easier to remember than uninteresting ones.
a. completely agree
b. agree
c. don't know
d. disagree
e. completely disagree

2. I am good at remembering names.
a. completely agree
b. agree
c. don't know
d. disagree
e. completely disagree

3. Do you keep a list or write down important dates, such as birthdays?
a. never
b. rarely
c. sometimes
d. often
e. always

4. Having a good memory is important to me.
a. completely agree
b. agree
c. don't know
d. disagree
e. completely disagree

5. I get upset when I can't remember something.

a. completely agree

b. agree

c. don't know

d. disagree

e. completely disagree
6. When you are looking for something you have recently misplaced, do you try to retrace your steps to find the object?
a. never
b. rarely
c. sometimes
d. often
e. always

7. I think having a good memory is a source of pride.
a. completely agree
b. agree
c. don't know
d. disagree
e. completely disagree

8. I find it more difficult to remember things when I am upset.
a. completely agree
b. agree
c. don't know
d. disagree
e. completely disagree

9. I am good at remembering birthdays.
a. completely agree
b. agree
c. don't know
d. disagree
e. completely disagree

10. I can remember things as well as I did in the past.
a. completely agree
b. agree
c. don't know
d. disagree
e. completely disagree 


\section{MSEQ em Português $1^{a}$. Página}

Estas questões são sobre a sua capacidade de memorizar nomes de pessoas. Para ajuda-lo(a) a decidir, considere a lista abaixo como exemplo: Rafael, Gabriela, Lucas, Laura, Felipe, Claudia, Marcelo, Susana, Marcos, Norma

1. Se alguém me mostrasse fotos de 10 pessoas e me dissesse seus nomes uma vez, eu conseguiria identificar as 10 pessoas por nome se eu visse as fotos novamente alguns minutos depois. (Marque NÃO ou SIM)
NÃO SIM
$10 \%$
$20 \quad 30$
$40 \quad 50$
$60 \quad 70$
80
$90 \quad 100 \%$

2.Se alguém me mostrasse fotos de 10 pessoas e me dissesse seus nomes uma vez, eu conseguiria identificar 8 pessoas por nome se eu visse as fotos novamente alguns minutos depois. (Marque NÃO ou SIM)
NÃO SIM
$10 \%$
$20 \quad 30$
40
50
$60 \quad 70$
80
$90 \quad 100 \%$

3.Se alguém me mostrasse fotos de 10 pessoas e me dissesse seus nomes uma vez, eu conseguiria identificar 6 pessoas por nome se eu visse as fotos novamente alguns minutos depois. (Marque NÃO ou SIM)
NÃO SIM
$10 \%$
20
30
$40 \quad 50$
$60 \quad 70$
80
$90 \quad 100 \%$

4.Se alguém me mostrasse fotos de 10 pessoas e me dissesse seus nomes uma vez, eu conseguiria identificar 4 pessoas por nome se eu visse as fotos novamente alguns minutos depois. (Marque NÃO ou SIM)
NÃO SIM
$10 \%$
20
30
40
50
$60 \quad 70$
80
$90 \quad 100 \%$

5.Se alguém me mostrasse fotos de 10 pessoas e me dissesse seus nomes uma vez, eu conseguiria identificar 2 pessoas por nome se eu visse as fotos novamente alguns minutos depois. (Marque NÃO ou SIM)
NÃO SIM
$10 \%$
$20 \quad 30$
40
50
$60 \quad 70$
80
90
$100 \%$

\section{MSEQ - Questionário Original 1ª́. página}

These questions ask about your ability to remember people's names. To help you, here is a sample list of names:

Melissa, James, Sarah, Derek, Rachel, Daniel, Karen, Patrick, Angela, Brian

1. If someone showed me the photographs of 10 people and told me their names once, I could identify 10 persons by name if I saw the pictures again a few minutes later. (circle NO or YES)
NO $\quad$ YES $\quad 10 \% \quad 20 \quad 30$
$40 \quad 50$
$60 \quad 70$
80
90
$100 \%$

2. If someone showed me the photographs of 10 people and told me their names once, I could identify 8 persons by name if I saw the pictures again a few minutes later. (circle NO or YES)

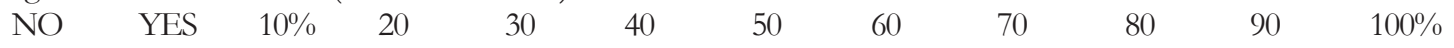

3.If someone showed me the photographs of 10 people and told me their names once, I could identify 6 persons by name if I saw the pictures again a few minutes later. (circle NO or YES)
$\mathrm{NO} \quad \mathrm{YES}$
$10 \%$
$20 \quad 30$
40
50
$60 \quad 70$
80
$90 \quad 100 \%$

4. If someone showed me the photographs of 10 people and told me their names once, I could identify 10 persons by name if I saw the pictures again a few minutes later. (circle NO or YES)
$\mathrm{NO}$
YES
$10 \% \quad 20$
30
$40 \quad 50$
60
70
80
$90 \quad 100 \%$

5. If someone showed me the photographs of 10 people and told me their names once, I could identify 10 persons by name if I saw the pictures again a few minutes later (circle NO or YES)
$\mathrm{NO}$
YES
$10 \%$
20
30
$40 \quad 50$
$60 \quad 70$
80
90
$100 \%$

\section{MSEQ - Retrotradução 1a. página}

These questions refer to your ability to memorize people's names. To help you decide, consider the list below as an example:

$$
\text { Rafael, Gabriela, Lucas, Laura, Felipe, Claudia, Marcelo, Susana, Marcos, Norma }
$$

1. If someone showed me the photos of 10 people and told me their names once, I would be able to identify the 10 people by name if I saw the photos again a few minutes later. (Circle NO or YES)
YES
$10 \%$
40
50
$60 \quad 70$
80
$90 \quad 100 \%$

$\mathrm{NO}$

photos again a few minutes later. (Circle NO or YES)
NO
YES
$10 \%$
20
30
40
50
$60 \quad 70$
80
90
$100 \%$

3. If someone showed me the photos of 10 people and told me their names once, I would be able to identify 6 people by name if I saw the photos again a few minutes later. (Circle NO or YES)
NO YES $\quad 10 \% \quad 20 \quad 30 \quad 40$
$50 \quad 60 \quad 70$
80
90
$100 \%$

4. If someone showed me the photos of 10 people and told me their names once, I would be able to identify 4 people by name if I saw the photos again a few minutes later. (Circle NO or YES)
NO
YES
$10 \% \quad 20$
30
$40 \quad 50$
$60 \quad 70$
80
90
$100 \%$

5.If someone showed me the photos of 10 people and told me their names once, I would be able to identify 2 people by name if I saw the photos again a few minutes later. (Circle NO or YES)
$\mathrm{NO}$
YES
$10 \%$
20
30
40
50
60
70
80
90
$100 \%$ 


\section{Anexo B}

Um exemplar das listas de supermercado

Lista A

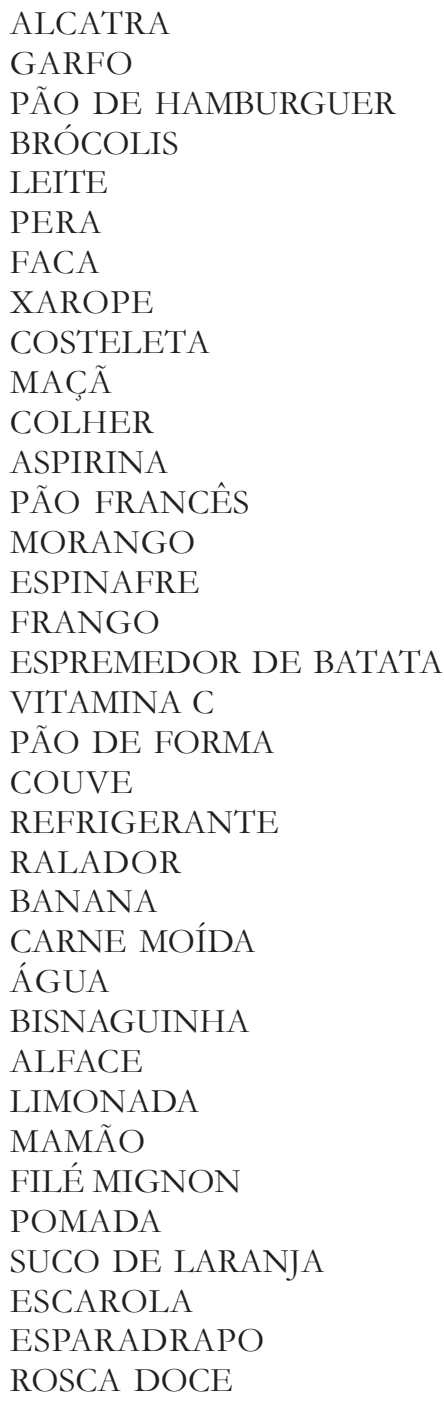

Lista A dividida em categorias

CARNES

ALCATRA

COSTELETA

FRANGO

CARNE MOÍDA

FILÉ MIGNON

$\underline{\text { FRUTAS }}$

PERA

MORANGO

BANANA

MAÇ $\tilde{A}$

MAMÃO

UTENSÍLIOS DE COZINHA

GARFO

FACA

COLHER

ESPREMEDOR DE BATATAS

RALADOR

$\underline{\mathrm{PAES}}$

PÃO DE HAMBURGUER

PÃO FRANCÊS

PÃO DE FORMA

BISNAGUINHA

ROSCA DOCE

VERDURAS/LEGUMES

BRÓCOLIS

ESCAROLA

ALFACE

COUVE

ESPINAFRE

LIQUIDOS

SUCO DE LARANJA

LEITE

LIMONADA

REFRIGERANTE

ÁGUA

MEDICAMENTOS

XAROPE

VITAMINA C

ESPARADRAPO

POMADA

ASPIRINA

\section{O visitante italiano}

Anexo C

Um exemplar das histórias curtas

Uma das experiências mais emocionantes na vida de Bernardo foi a visita surpresa de seu primo italiano. Bernardo sabia que seu pai tinha imigrado para o Brasil por volta de 1910. Separadamente, sua mãe também veio na mesma época. Eles se conheceram e se casaram no Brás, em São Paulo. Ambos contavam histórias de irmãos e irmãs que haviam deixado. Bernardo até mesmo se lembrava de ver seus pais escrevendo e mandando pacotes para os parentes italianos. Mas de acordo com sua lembrança, o último contato aconteceu nos anos 50. Bernardo sabia que tinha primos na Itália, mas até esta visita, ele nunca tinha conhecido nenhum. Era uma tarde tranqüila de sábado, quando Pietro bateu a sua porta. Pelo sotaque e pelas roupas, Bernardo logo percebeu que ele era italiano. Pietro explicou que estava em São Paulo a passeio, mas um dos seus objetivos era encontrar seus parentes brasileiros. Ele só sabia o sobrenome e que moravam no Brás. 